\title{
On the Outer-Independent Double Roman Domination of Graphs
}

\author{
Yongsheng Rao ${ }^{1}$, Saeed Kosari ${ }^{1 *}$, Seyed Mahmoud Sheikholeslami ${ }^{2}$, M. Chellali $^{3}$ and \\ Mahla Kheibari ${ }^{2}$
}

${ }^{1}$ Institute of Computing Science and Technology, Guangzhou University, Guangzhou, China, ${ }^{2}$ Department of Mathematics, Azarbaijan Shahid Madani University, Tabriz, Iran, ${ }^{3}$ LAMDA-RO Laboratory, Department of Mathematics, University of Blida, Blida, Algeria

OPEN ACCESS

Edited by:

Yong Chen,

Hangzhou Dianzi University, China

Reviewed by:

Zepeng Li,

Lanzhou University, China

Sarfraz Ahmad,

COMSATS University Islamabad,

Pakistan

*Correspondence:

Saeed Kosar

saeedkosari38@yahoo.com

Specialty section:

This article was submitted to

Optimization,

a section of the journal

Frontiers in Applied Mathematics

and Statistics.

Received: 09 November 2020 Accepted: 04 December 2020

Published: 05 February 2021

Citation:

Rao Y, Kosari S, Sheikholeslami SM, Chellali $M$ and Kheibari M (2021) On the Outer-Independent Double Roman

Domination of Graphs.

Front. Appl. Math. Stat. 6:559132.

doi: 10.3389/fams.2020.559132
An outer-independent double Roman dominating function (OIDRDF) of a graph $G$ is a function $h: V(G) \rightarrow\{0,1,2,3\}$ such that i) every vertex $v$ with $f(v)=0$ is adjacent to at least one vertex with label 3 or to at least two vertices with label 2 , ii) every vertex $v$ with $f(v)=1$ is adjacent to at least one vertex with label greater than 1, and iii) all vertices labeled by 0 are an independent set. The weight of an OIDRDF is the sum of its function values over all vertices. The outer-independent double Roman domination number $\gamma_{\text {oidR }}(G)$ is the minimum weight of an OIDRDF on $G$. It has been shown that for any tree $T$ of order $n \geq 3, \gamma_{\text {oidR }}(T) \leq 5 \mathrm{n} / 4$ and the problem of characterizing those trees attaining equality was raised. In this article, we solve this problem and we give additional bounds on the outerindependent double Roman domination number. In particular, we show that, for any connected graph $G$ of order $n$ with minimum degree at least two in which the set of vertices with degree at least three is independent, $\gamma_{\text {oidR }}(T) \leq 4 \mathrm{n} / 3$.

Keywords: outer independence double Roman domination, outer-independent double Roman dominating function, independent set, double Roman domination, Roman domination, tree

\section{INTRODUCTION}

We consider only simple connected graphs $G$ with vertex set $V=V(G)$ and edge set $E=E(G)$, where $n=|V|$ is the order of $G$. The open neighborhood of a vertex $v \in V$ is the set $N(v)=\{u \mid u v \in E\}$, and the degree of $v$ is $\operatorname{deg}_{G}(v)=|N(v)|$. A leaf is a vertex with degree one and its neighbor is called a stem. A strong stem is a stem adjacent to at least two leaves. The diameter of $G$, denoted by diam $(G)$, is the maximum value among distances between all pairs of vertices of $G$.

A set $S \subset V$ is independent if no two vertices in $S$ are adjacent. The independence number $\alpha(G)$ of a graph $G$ is the maximum cardinality among the independent sets of vertices of $G$. A vertex cover of a graph $G$ is a set of vertices such that each edge of the graph is incident to at least one vertex of the set. A minimum vertex cover is a vertex cover of smallest possible size. The vertex cover number $\alpha_{0}(G)$ is the minimum cardinality of a vertex cover of $G$.

In 2016, Beeler et al., Ref. 1, introduced the concept of double Roman domination and defined a double Roman dominating function (DRDF) on a graph $G$ to be a function $h: V(G) \rightarrow\{0,1,2,3\}$ such that each vertex with label 0 is adjacent to a vertex labeled 3 or to at least two vertices labeled 2 , and each vertex with label 1 is adjacent to a vertex labeled 2 or 3 . The weight of a DRDF $f$ is the value $h[V(G)]=\sum_{u \in V(G)} h(u)$, and the double Roman domination number $\gamma_{d R}(G)$ equals the minimum weight of a DRDF on $G$. Double Roman domination has been studied by several authors; see, for example, Refs. 2-14. For more details on Roman domination and its variations, we refer the reader to Refs. 15-18. 
For double Roman domination, one can think of any vertex representing a location in the Roman Empire and any edge being a road between two locations. A location is said to be protected if at least one army is stationed in it or by sending to it two armies from neighboring location(s) having already more than two armies (according to the decree of Emperor Constantine the Great). A locality without an army is certainly vulnerable, and it will be even more vulnerable if one of its neighbors is without army too. Hence, the best situation for a location with no army is to be surrounded by locations where each with at least one army. This leads us to seek an DRDF $h=\left(V_{0}, V_{1}, V_{2}, V_{3}\right)$, where $V_{0}$ is an independent set; that is, $h$ is an OIDRDF.

Regarding this, Abdollahzadeh Ahangar et al., Ref. 19, introduced a new variation of double Roman domination called outer-independent double Roman domination. An outerindependent double Roman dominating function (OIDRDfunction) of a graph $G$ is a DRDF $h$ such that the set of vertices assigned a 0 under $h$ is independent. The outer-independent double Roman domination number (OIDRD-number for short) $\gamma_{\text {oidR }}(G)$ is the minimum weight of an OIDRD-function on G. Clearly, $\gamma_{d R}$ $(G) \leq \gamma_{\text {oidR }}(G)$ holds for every graph $G$. Recently, Mojdeh et al., Ref. 20 , proved that the decision problem associated with $\gamma_{\text {oidR }}(G)$ is NP-complete even when restricted to planar graphs with maximum degree at most four. They also characterized the families of all connected graphs with small outer-independent double Roman domination numbers.

In the following, we denote the set $\{0,1,2,3\}$ by [3].

The authors of Ref. 19 provided an upper bound for the OIDRD-number of trees in terms of the order and number of stems.

Theorem 1.1. For each tree $\mathrm{T}$ on $n \geq 3$ vertices,

$$
\gamma_{\text {oidR }}(T) \leq n+\frac{s(T)}{2}
$$

where $s(T)$ is the number of stems of $T$.

Since the number of stems of any tree does not exceed half the order of the tree, the next result is immediate from Theorem 1.1.

Proposition 1.2. For every tree $T$ on $n \geq 4$ vertices, $\gamma_{\text {oidR }}$ $(T) \leq 5 \mathrm{n} / 4$.

Moreover, it should be noted that the problem of characterizing the trees $T$ attaining equality in the upper bound of Proposition 1.2 was raised in Ref. 19. This problem will be solved in this article, and additional bounds on the OIDRD-number will be given. In particular, we prove that, for any connected graph $G$ of order $n$ with minimum degree at least two in which the set of vertices with degree at least three is independent, $\gamma_{\text {oidR }}(G) \leq 4 n / 3$.

\section{TREES T OF ORDER $n$ WITH $\gamma_{\text {oidR }}(T)=5 n / 4$}

With the aim of characterizing the trees $T$ of order $n \geq 3$ with $\gamma_{\text {oidR }}$ $(T)=5 n / 4$, let $\mathcal{T}_{t}$ be the family of trees defined as follows. Let $H_{i}$ be a path $P_{4}$ whose vertices are labeled in order $v_{1}^{i}, v_{2}^{i}, v_{3}^{i}$, and $v_{4}^{i}$. For any integer $t \geq 1$, let $\mathcal{T}_{t}$ be the family of trees $T$ obtained from $H_{1}, \ldots, H_{t}$ by adding $t-1$ edges between the stems of $H_{i}$ 's so that the resulting graph is a tree. Beeler et al., Ref. 1, proved that, for every tree $T \in \mathcal{T}_{t}$, $\gamma_{d R}(T)=5|V(T)| / 4$ which implies that $\gamma_{\text {oidR }}(T)=5|V(T)| / 4$.

Lemma 2.1. Let $T \in \mathcal{T}_{t}$ for some integer $t \geq 1$. Then, there is a $\gamma_{\text {oidR }}(T)$ function $f$ such that, for every leaf $v$ of $T, f(v) \in\{1,2\}$.

Proof. Let $T$ be a tree of $\mathcal{T}_{t}$ for some integer $t \geq 1$. Then, $\gamma_{\text {oidR }}(T)=5|V(T)| / 4$. Since $T$ is a bipartite graph, let $X$ and $Y$ be the partite sets of $T$. Let $X^{\prime}$ be the set of stems of $T$ belonging to $X$, and likewise let $Y^{\prime}$ be defined similarly. Clearly, $|X|=|Y|$, $\left|X^{\prime}\right|=\left|Y^{\prime}\right|$, and every leaf of $T$ is either in $X-X^{\prime}$ or $Y-Y^{\prime}$. Define the function $f$ on $V(T)$ by assigning a 2 to all vertices of $Y$, a 1 to all vertices in $X-X^{\prime}$, and a 0 to vertices in $X$. Then, $f$ is an OIDRD-function of $T$ of weight $5|V(T)| / 4$, and thus $f$ is a $\gamma_{\text {oidR }}$ (T) function with desired property. $\square$

Theorem 2.2. Let $T$ be a tree on $n \geq 4$ vertices. Then, $\gamma_{\text {oidR }}(T)=$ $5 n / 4$ if and only if $T \in \mathcal{T}_{t}$ for some integer $t \geq 1$.

Proof. We prove only the necessity. Let $T$ be a tree of $n \geq 4$ such that $\gamma_{\text {oidR }}(T)=5 n / 4$. Clearly, $n=4 t$ for some integer $t \geq 1$. To prove that $T \in T_{t}$, we use an induction on $t$. If $t=1$, then $T=P_{4}$, and clearly, $T \in \mathcal{T}_{1}$. Let $t \geq 2$ and assume that the result is true for any tree $T$ with $\gamma_{\text {oidR }}(T)=5 n / 4$, where $n=4 t^{\prime}$ and $t^{\prime}<t$. Let $T$ be a tree with $\gamma_{o i d R}(T)=5 n / 4$ and $n=4 t$. We deduce from $\gamma_{\text {oidR }}(T)=5 n / 4 \leq n+s(T) / 2 \leq 5 n / 4$ that $s(T)=n / 2$. Therefore, $T$ is the corona of some tree and so $T$ has no strong stem. Moreover, $\operatorname{diam}(T) \geq 4$ because $t \geq 2$. Let $P=v_{1} v_{2}, \ldots, v_{k}$ be a diametral path in $T$ and root $T$ at $v_{k}$. Then, $\operatorname{deg}_{T}\left(v_{2}\right)=2$, and there is a unique leaf $w$ adjacent to $v_{3}$. Denote by $T_{x}$ the subtree induced by a vertex $x$ and its descendants in the rooted tree $T$. We claim that $\operatorname{deg}_{T}\left(v_{3}\right)=3$. Suppose, to the contrary, that $\operatorname{deg}_{T}\left(v_{3}\right) \geq 4$ and let $v_{2}=w_{1}, w_{2}, \ldots, w_{s}$ be the children of $v_{3}$ with depth one. Since $\operatorname{deg}_{T}\left(w_{i}\right)=2$ for each $i$, let $w_{i}^{\prime}$ be the leaf adjacent to $w_{i}$ for $i \in\{2, \ldots, s\}$, and consider the tree $T^{\prime}$ obtained from $T$ by removing every $w_{i}$ and $w_{i}^{\prime}$ for $i \geq 2$. Observe that the subtree rooted at $v_{3}$ is a path $P_{4}$. Let $f$ be a $\gamma_{\text {oidR }}\left(T^{\prime}\right)$-function, where, without loss of generality, $f\left(v_{3}\right)=3$. Then, $f$ can be extended to an OIDRD-function of $T$ by assigning a 0 to $w_{2}, \ldots, w_{s}$ and a 2 to $w_{2}^{\prime}, \ldots, w_{s}^{\prime}$. It follows from Proposition 1.2 that

$$
\gamma_{o i d R}(T) \leq \gamma_{o i d R}\left(T^{\prime}\right)+2(s-1) \leq \frac{5(n-2 s+2)}{4}+2 s-2<\frac{5 n}{4},
$$

which leads to a contradiction. Hence, $\operatorname{deg}_{T}\left(v_{3}\right)=3$, and thus $T_{v_{3}}=P_{4}$. Now, let $T^{\prime \prime}=T-T_{v_{3}}$. Clearly, any $\gamma_{\text {oidR }}\left(T^{\prime \prime}\right)$-function $f$ can be extended to an OIDRD-function of T by assigning a 3 to $v_{3}$, 2 to $v_{1}$, and a 0 to $v_{2}, w$. Hence,

$$
\frac{5 n}{4}=\gamma_{o i d R}(T) \leq \gamma_{o i d R}\left(T^{\prime \prime}\right)+5 \leq \frac{5(n-4)}{4}+5=\frac{5 n}{4} .
$$

Therefore, by the induction hypothesis on $T^{\prime \prime}$, we have $T^{\prime \prime} \in \mathcal{T}_{n / 4-1}$. By the construction of $T^{\prime \prime}$, we may assume that $v_{4}$ is a vertex of an induced path $\quad P_{4}=v_{1}^{1} v_{2}^{1} v_{3}^{1} v_{4}^{1}, \quad$ where $\quad \operatorname{deg}_{T^{\prime \prime}}\left(v_{1}^{1}\right)=\operatorname{deg}_{T^{\prime \prime}}\left(v_{4}^{1}\right)=1$, $\operatorname{deg}_{T^{\prime \prime}}\left(v_{3}^{1}\right)=2$, and $\operatorname{deg}_{T^{\prime \prime}}\left(v_{2}^{1}\right) \geq 2$. Now, if $n / 4-1=1$, then clearly $T \in \mathcal{T}_{2}$, and we are done. Hence, we assume that $n / 4-1 \geq 2$. We claim that $v_{4} \in\left\{v_{2}^{1}, v_{3}^{1}\right\}$. Suppose, to the contrary, that $v_{4} \in\left\{v_{1}^{1}, v_{4}^{1}\right\}$. By Lemma 2.1, there is a $\gamma_{\text {oidR }}\left(T^{\prime \prime}\right)$-function $f$ such that $f\left(v_{4}\right) \in\{1,2\}$. If $f\left(v_{4}\right)=1$, then $f\left(v_{5}\right)=2$ and the function $h$ defined on $V(T)$ by $h\left(v_{3}\right)=3, h\left(v_{1}\right)=2, h(w)=h\left(v_{2}\right)=h\left(v_{4}\right)=0$, and $h(x)=f(x)$ 
for $x \in V\left(T^{\prime \prime}\right)-\left\{v_{4}\right\}$ is an OIDRD-function of $T$ of weight $\omega\left(h_{1}\right)+4=5(n-4) / 4+4<5 n / 4$, a contradiction. If $f\left(v_{4}\right)=2$ and $f\left(v_{5}\right) \geq 1$, then as above we can get a contradiction. Hence, assume that $f\left(v_{4}\right)=2$ and $f\left(v_{5}\right)=0$. Then, $v_{5}$ has a neighbor with weight at least two and the function $h^{\prime}$ defined on $V(T)$ by $h^{\prime}\left(v_{3}\right)=3, h^{\prime}\left(v_{1}\right)=2, \quad h^{\prime}\left(v_{5}\right)=1, \quad h^{\prime}\left(v_{4}\right)=h^{\prime}(w)=h^{\prime}\left(v_{2}\right)=0$, and $h^{\prime}(x)=f(x)$ for $x \in V\left(T^{\prime \prime}\right)-\left\{v_{4}, v_{5}\right\}$ is an OIDRD-function of $T$ of weight $\omega\left(h^{\prime}\right)+4=5(n-4) / 4+4<5 n / 4$, a contradiction. Thus, $v_{4} \in\left\{v_{2}^{1}, v_{3}^{1}\right\}$, and thus $T \in \mathcal{T}_{t}$ as desired. This completes the proof. $\square$

\section{SLIGHTLY IMPROVED BOUNDS FOR TREES}

In this section, we present some sharp bounds on the OIDRDnumber. We start with some classes of trees where the upper bound in Proposition 1.2 will be slightly improved.

Proposition 3.1. Let $T$ be a tree of order $n \geq 3$, where $n \equiv 0,1,2(\bmod 4)$. If $T$ contains a strong stem, then $\gamma_{\text {oidR }}(T) \leq$ $5 \mathrm{n} / 4-1$.

Proof. Let $s \in V(T)$ be a strong stem of $T$ and let $L(s)=$ $\left\{x_{1}, x_{2}, \ldots, x_{t}\right\}$ be the set of leaves adjacent to $s$. Consider the forest $T-s$ and assume that it contains $r \geq 0$ components each of order at least four $T_{1}, \ldots, T_{r}$. If $r \geq 1$, then $f_{i}$ be a $\gamma_{\text {oidR }}\left(T_{i}\right)$ function for each $i \in\{1, \ldots r\}$. Clearly, by Proposition 1.2, $\gamma_{\text {oidR }}\left(T_{i}\right) \leq 5\left|V\left(T_{i}\right)\right| / 4$. Moreover, suppose that $T-s$ has $t_{2}$ components isomorphic to $K_{2}$ and $t_{3}$ components isomorphic to $P_{3}$. Clearly, all $f_{i}$ 's together can be extended to an OIDRDfunction to $T$ by assigning a 3 to $s$ and to all center vertices of the components of order three, a 2 to each leaf at distance two from $s$ belonging to a component of order two, and a 0 to the remaining vertices in the components of order at most three. Observe that if $r=0$, then the total weight assigned to the vertices is at most $p$, and thus $\gamma_{o i d R}(T) \leq n<5 n / 4$. Hence, we can assume that $r \geq 1$. Now, using the fact that $n \equiv 0,1,2(\bmod 4)$, we obtain

$$
\begin{aligned}
\gamma_{\text {oidR }}(T) & \leq 3+2 t_{2}+3 t_{3}+\sum_{i=1}^{r} \frac{5\left|V\left(T_{i}\right)\right|}{4} \\
& =3+2 t_{2}+3 t_{3}+\frac{5\left(n-3 t_{3}-2 t_{2}-1-t\right)}{4} \\
& \leq 5 n / 4-1,
\end{aligned}
$$

as desired. $\square$

A closer look at the proof of Proposition 3.1 shows that it can be used to obtain the next two results too.

Proposition 3.2. Let $T$ be a tree of order $n \geq 3$, where $n \equiv 3(\bmod 4)$. If $T$ contains one strong stem such that $T-s$ contains a component isomorphic to $P_{2}$ or $P_{3}$, then $\gamma_{\text {oidR }}(T) \leq 5 n / 4-1$.

Proposition 3.3. Let $T$ be a tree of order $n \geq 3$, where $n \equiv 3(\bmod 4)$. If $T$ contains a strong stem having at least three leaves, then $\gamma_{\text {oidR }}(T) \leq 5 n / 4-1$.

Proposition 3.4. Let $T$ be a tree of order $n \geq 3$, where $n \equiv 3(\bmod 4)$. If $T$ contains more than one strong stem, then $\gamma_{\text {oidR }}(T) \leq 5 n / 4-1$.

Proof. Let $s_{1}$ and $s_{2}$ be two strong stems of $T$ and let $L\left(s_{i}\right)=\left\{x_{1}^{i}, x_{2}^{i}, \ldots, x_{t_{i}}^{i}\right\}$ be the set of leaves adjacent to $s_{i}$ for $i \in\{1,2\}$. Clearly, $\operatorname{diam}(T) \geq 3$. If each component of $T-$ $\left\{s_{1}, s_{2}\right\}$ is of order at most three, then assigning a 3 to $s_{1}, s_{2}$ and to the center vertex of each component of order three, a 2 to each leaf at distance two from $s_{i}$ that belongs to a component of order two in $T-\left\{s_{1}, s_{2}\right\}$, and a 0 to the remaining vertices provides an OIDRD-function of $T$ of weight at most $p$. Therefore, $\gamma_{\text {oidR }}(T) \leq n \leq 5 n / 4-1$. Hence, we may assume that $T-s$ contains at least one component of order at least four. Let $T_{1}, \ldots, T_{r}(r \geq 1)$ be such components of $T-\left\{s_{1}, s_{2}\right\}$ of order at least four. Let $f_{i}$ be a $\gamma_{\text {oidR }}\left(T_{i}\right)$ function for each $i$. In addition, let $T-\left\{s_{1}, s_{2}\right\}$ have $t_{2}$ components isomorphic to $K_{2}$ and $t_{3}$ components isomorphic to $P_{3}$. Then, all $f_{i}$ 's together can be extended to an OIDRD-function of $T$ by assigning a 3 to $s_{1}, s_{2}$ and to the center vertex of each component of order three, a 2 to each leaf at distance two from $s$ that belongs to a component of order two in $T-\left\{s_{1}, s_{2}\right\}$, a 3 to the center of all components of order three, $f_{i}(x)$ for each $x \in V\left(T_{i}\right)$ and $i \in\{1, \ldots, r\}$, and a 0 to the remaining vertices in the components of order at most three. Using the fact that $n \equiv 3(\bmod 4)$, we obtain

$$
\begin{aligned}
\gamma_{o i d R}(T) & \leq 6+2 t_{2}+3 t_{3}+\sum_{i=1}^{r} \frac{5\left|V\left(T_{i}\right)\right|}{4} \\
& =6+2 t_{2}+3 t_{3}+\frac{5\left(n-3 t_{3}-2 t_{2}-2-t_{1}-t_{2}\right)}{4} \\
& \leq 5 n / 4-1,
\end{aligned}
$$

as desired. $\square$

\section{GRAPHS WITH MINIMUM DEGREE TWO}

We begin by recalling the question, posed in Ref. 19, on whether the $5 n / 4$ upper bound on the OIDRD-number for trees remains valid for arbitrary graphs. In this section, we restrict our attention to graphs with minimum degree at least two such that the set of vertices of degree at least three is independent set, where we shall show that the OIDRDnumber is bounded above by $4 n / 3$. We will use the following result established in Ref. 19.

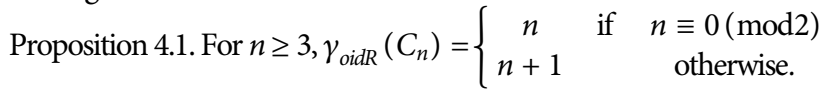

Proposition 4.2. For $n \geq 3$, the path $P_{n}$ has an OIDRD-function $f$ that assigns positive weight to the end-vertices of $P_{n}$ and $\omega(f)=n+1 \leq 4 n / 3$.

Proof. Let $P_{n}=v_{1} v_{2} \ldots v_{n}$ and define the function $f$ on $V\left(P_{n}\right)$ as follows. If $n \equiv 1(\bmod 2)$, then $f\left(v_{2 i+1}\right)=2$ for $0 \leq i \leq n-1 / 2$ and $f(x)=0$ otherwise, and if $n \equiv 0(\bmod 2)$, then $f\left(v_{n}\right)=1$, $f\left(v_{2 i+1}\right)=2$ for $0 \leq i \leq n-2 / 2$ and $f(x)=0$ otherwise. Clearly, $\omega(f) \leq 4 n / 3$ and $f$ is an OIDRD-function of $P_{n}$ assigning positive weight to the end-vertices of $P_{n}$. $\square$

For integers $r \geq 3$ and $s \geq 1$, let $C_{r, s}$ be the graph obtained from a cycle $C_{r}=\left(u_{1} u_{2} \ldots u_{r}\right)$ and a path $P_{s}=v_{1} v_{2} \ldots v_{s}$ by adding the edge $u_{1} v_{1}$. Applying Propositions 4.1 and 4.2 , we derive the next result. 
Proposition 4.3. For integers $r \geq 3$ and $s \geq 1$, the graph $C_{r, s}$ has an OIDRD-function $f$ that assigns a positive weight to $v_{s}$ and $\omega(f) \leq$ $4(r+s) / 3$.

Proof. Assume first that $r+s \in\{4,5\}$. If $r+s=4$, then assigning a 1 to $v_{1}$, a 2 to $u_{1}, u_{2}$, and a 0 to $u_{3}$ provides an OIDRD-function of $C_{3,1}$ satisfying the conditions. If $r=4$ and $s=1$, then assigning a 1 to $v_{1}$, a 2 to $u_{1}, u_{3}$, and a 0 to $u_{2}$ and $u_{4}$ provides an OIDRD-function of $C_{4,1}$ with the desired properties. If $r=3$ and $s=2$, then assigning a 2 to $u_{1}, u_{3}, v_{2}$ and a 0 to $u_{2}$ and $v_{1}$ provides as above an OIDRD-function of $C_{3,2}$ with the desired properties. Hence, assume that $r+s \geq 6$, and let $f$ be a $\gamma_{\text {oidR }}\left(C_{r}\right)$ function such that $f\left(u_{1}\right) \geq 2$. If $s=1$, then the function $g$ defined on $V\left(C_{r, s}\right)$ by $g\left(v_{1}\right)=1$ and $g(x)=f(x)$ otherwise is an OIDRDfunction of $C_{r, s}$ with desired properties. If $s=2$, then the function $g$ defined on $V\left(C_{r, s}\right)$ by $g\left(v_{1}\right)=0, g\left(v_{2}\right)=2$, and $g(x)=f(x)$ otherwise is an OIDRD-function of $C_{r, s}$ with the desired properties. Henceforth, we can assume that $s \geq 3$.

Let $g$ be an OIDRD-function of the path $v_{1} v_{2} \ldots v_{s}$ of weight $s+1$ assigning positive weights to $v_{1}, v_{s}$ (Proposition 4.2). Define $h$ on $C_{r, s}$ by $h\left(u_{i}\right)=f\left(u_{i}\right)$ for each $i$ and $h\left(v_{j}\right)=g\left(v_{j}\right)$ for each $\mathrm{j}$. Clearly, $h$ is an OIDRD-function of $C_{r, s}$ and we deduce from Proposition 4.1 that $\gamma_{\text {oidR }}\left(C_{r, s}\right) \leq r+s+2 \leq 4(r+s) / 3$. $\square$

Let $\mathcal{F}$ be the family of all simple graphs obtained from some connected multigraph $H$ without loops with $\delta(H) \geq 3$ by subdividing each edge of $H$ at least once and at most five times. Clearly, any graph in $\mathcal{F}$ has order at least 5 . The next result shows that every graph $G$ in $\mathcal{F}$ of order $n$ satisfies $\gamma_{\text {oidR }}(G) \leq 4 n / 3$.

Proposition 4.4. For any graph $G \in \mathcal{F}$ of order $n$, there exists an OIDRD-function fof $G$ such that $\omega(f) \leq 4 n / 3$ and $f(x) \geq 2$ for each vertex $x$ of degree at least three.

Proof. Let $G \in \mathcal{F}$ be a graph of order $n$. We use an induction on $n$. If $n=5$, then $G=K_{2,3}$ and the function $f$ that assigns a 2 to the vertices of degree 3 and a 0 to the remaining vertices satisfies the conditions as desired. Let $n \geq 6$ and assume that the result holds for all graphs in $\mathcal{F}$ of order $n^{\prime}$, where $5 \leq n^{\prime}<n$. Let $G \in \mathcal{F}$ be a graph of order $n \geq 6$. Suppose that $A=\left\{x \in V(G) \mid \operatorname{deg}_{G}(x) \geq 3\right\}$ and let $B=V(G)-A$. In the sequel, we will call an induced path $P$ of $G$ an $A$-ear path if $V(P) \subset B$ and $P$ is connected to $A$ by either its unique vertex (when $|V(P)|=1$ ) or its two end-vertices (when $|V(P)| \geq 2$ ). For each $i \in\{1,2,3,4,5\}$, let $Q_{i}$ be the set of all $A$-ear paths $P$ of $G$ of order $i$ and let $\mathcal{Q}=\cup_{i=1}^{5} Q_{i}$. Clearly, $B=\cup_{P \in \mathcal{Q}} V(P)$. Moreover, for each $A$-ear path $P$, let $X_{P}=\{u \in A \mid u$ is adjacent to a vertex of $P\}$. Hence, $A=\cup_{P \in \mathcal{Q}} X_{P}$. Furthermore, since $G \in \mathcal{F}$, we have $\left|X_{P}\right|=2$ for each $P \in \mathcal{Q}$, and therefore, $|A| \geq 2$.

First, let $Q_{3} \cup Q_{5} \neq \varnothing$. Suppose $P=x_{1} \ldots x_{2 k+1} \in Q_{2 k+1}(k \in\{1,2\})$ and let $X_{P}=\left\{a_{1}, a_{2}\right\}$, where $a_{1} x_{1}, a_{2} x_{2 k+1} \in E(G)$. Let $G^{\prime}$ be the graph obtained from $G$ by first removing all vertices of the path $P$ except $x_{k+1}$ and then adding edges $a_{1} x_{k+1}$ and $a_{2} x_{k+1}$. Clearly, $G^{\prime} \in \mathcal{F}$ of order less than $n$. By the induction hypothesis on $G^{\prime}$, there exists an OIDRD-function $f=\left(V_{0}, V_{1}, V_{2}, V_{3}\right)$ of $G^{\prime}$ such that $a_{1}, a_{2} \in V_{2} \cup V_{3}$ and $\omega(f) \leq 4(n-2 k) / 3$. It follows that $f\left(x_{k+1}\right)=0$. Now, if $k=1$, then the function $g$ defined on $V(G)$ by $g\left(x_{2}\right)=2, g\left(x_{1}\right)=g\left(x_{3}\right)=0$, and $g(x)=f(x)$ otherwise is an OIDRD-function of $G$ such that $g(x) \geq 2$ for each $x \in A$ and

$$
\omega(g)=\omega(f)+2 \leq \frac{4(n-2)}{3}+2<\frac{4 n}{3} .
$$

If $k=2$, then the function $g$ defined on $V(G)$ by $g\left(x_{2}\right)=g\left(x_{4}\right)=2$, $g\left(x_{1}\right)=g\left(x_{3}\right)=g\left(x_{5}\right)=0$, and $g(x)=f(x)$ otherwise is an OIDRD-function of $G$ such that $g(x) \geq 2$ for each $x \in A$ and

$$
\omega(g)=\omega(f)+4 \leq \frac{4(n-4)}{3}+4<\frac{4 n}{3} .
$$

From now on, we can assume that $Q_{3} \cup Q_{5}=\varnothing$.

Next, assume that $Q_{4} \neq \varnothing$ and let $P=x_{1} x_{2} x_{3} x_{4} \in Q_{4}$ with $X_{P}=$ $\{u, a\}$ and $u x_{1}, a x_{4} \in E(G)$. Let $G^{\prime}$ be the graph obtained from $G$ by deleting $x_{1}, x_{2}, x_{3}$ and adding the edge $u x_{4}$. Clearly, $G^{\prime} \in \mathcal{F}$ and thus by the induction hypothesis, there exists an OIDRDfunction $f$ of $G^{\prime}$ such that $f(x) \geq 2$ for each vertex $x \in A$ and $\omega(f) \leq 4(n-3) / 3$. Then, the function $g$ defined on $V(G)$ by $g\left(x_{2}\right)=g\left(x_{3}\right)=2, g\left(x_{1}\right)=0$, and $g(x)=f(x)$ otherwise is an OIDRD-function of $G$ such that $g(x) \geq 2$ for each $x \in A$ and $\omega(g)=\omega(f)+4 \leq 4(n-3) / 3+4=4 n / 3$.

Considering the above situations, we may assume that $\mathcal{Q}=Q_{1} \cup Q_{2}$. Note that $n=|A|+m_{1}+2 m_{2}$ and $m_{1}+m_{2} \geq 3$, where $m_{i}=\left|Q_{i}\right|$ for $i \in\{1,2\}$.

Assume first that $|A|=2$ and let $A=\{u, v\}$. If $Q_{2} \neq \varnothing$, then let $Q_{2}=\left\{w_{1}^{j} w_{2}^{j} \mid 1 \leq j \leq m_{2}\right\}$, where $u w_{1}^{j} \in E(G)$ for each $j$. Moreover, if $Q_{1} \neq \varnothing$, then let $Q_{1}=\left\{z_{1}^{l} \mid 1 \leq l \leq m_{1}\right\}$. Define the function $g$ on $V(G)$ by $g(u)=3, g(v)=2, g\left(w_{2}^{j}\right)=1$ for each $j$ and $g(t)=0$ otherwise. Then, $g$ is an OIDRD-function of $G$ such that $g(x) \geq 2$ for each $x \in A$ and we have $\omega(g) \leq 5+m_{2} \leq 4\left(2+2 m_{2}+m_{1}\right) / 3=4 n / 3$.

Henceforth, we can assume that $|A| \geq 3$. We consider the following cases.

Case 1. $Q_{2}=\varnothing$.

Then, $\mathcal{Q}=Q_{1}$ and $G$ is obtained from a loopless multigraph $G^{\prime}$ by subdividing each edge of $G^{\prime}$ once. Since each vertex of $G^{\prime}$ has degree at least three, we have $2\left|E\left(G^{\prime}\right)\right| \geq 3 n\left(G^{\prime}\right)$ and so $n(G)=\left|V\left(G^{\prime}\right)\right|+\left|E\left(G^{\prime}\right)\right| \geq 5 n\left(G^{\prime}\right) / 2$. Define $f$ on $V(G)$ by $f(x)=$ 2 for $x \in V\left(G^{\prime}\right)$ and $f(x)=0$ otherwise. Clearly, $f$ is an OIDRfunction of $G$ such that $f(x) \geq 2$ for each $x \in A$ and $\omega(f)=$ $2 n\left(G^{\prime}\right) \leq 4 n / 3$.

Case 2. $Q_{2} \neq \varnothing$.

Let $u \in V(G)$ be a vertex with the most neighbors in $A$-ear paths of $Q_{2}$. We consider the following subcases.

Subcase 2.1. $u$ is adjacent to at least two $A$-ear paths of $Q_{2}$.

Let $P_{1}=x_{1} x_{2}, P_{2}=y_{1} y_{2} \in Q_{2}$ be two $A$-ear paths such that $u x_{1}, u y_{1} \in E(G)$. Assume that $\left\{a x_{2}, b y_{2}\right\} \subseteq E(G)$, where $a, b \in A-\{u\}$. Suppose that $a \neq b$, and let $G^{\prime}$ be the graph obtained from $G$ by removing first $u, x_{1}, x_{2}$ and then adding the edge $y_{1} a$ and joining by an edge every vertex $x$ in $N(u)-\left\{x_{1}, y_{1}\right\}$ to either $a$ or $b$ provided $a$ or $b$ is not adjacent to the end-vertex of the $A$-ear path containing $x$. Clearly, $G^{\prime} \in \mathcal{F}$, and by the induction hypothesis, there exists an OIDRD-function $f=\left(V_{0}, V_{1}, V_{2}, V_{3}\right)$ of $G^{\prime}$ such that $A-\{u\} \subseteq V_{2} \cup V_{3}$ and $\omega(f) \leq 4(n-3) / 3$. Define the function $g$ on $V(G)$ by $g(u)=3, g\left(x_{2}\right)=1, g\left(x_{1}\right)=0$, and $g(x)=$ $f(x)$ otherwise. Then, $g$ is an OIDR-function of $G$ such that $g(x) \geq 2$ for each $x \in A$ and $\omega(g)=\omega(f)+4 \leq 4(n-3) / 3+4=4 n / 3$.

Suppose now that $a=b$ and let $w \in A-\{u, a\}$. Let $G^{\prime}$ be the graph obtained from $G$ by removing first $u, x_{1}, x_{2}$ and then adding the edge $y_{1} w$ and joining every vertex $x$ in $N(u)-\left\{x_{1}, y_{1}\right\}$ to either $a$ or $w$ provided $a$ or $w$ is not adjacent to the end-vertex of the $A$-ear 
path containing $x$. Clearly, $G^{\prime} \in \mathcal{F}$, and thus by the induction hypothesis, there exists an OIDRD-function $f=\left(V_{0}, V_{1}, V_{2}, V_{3}\right)$ of $G^{\prime}$ such that $A-\{u\} \subseteq V_{2} \cup V_{3}$ and $\omega(f) \leq 4(n-3) / 3$. Now, the function $g$ defined above satisfies the desired conditions.

Subcase 2.2. All neighbors of $u$ but one belong to $A$-ear paths of $Q_{1}$.

By the choice of $u$, we may assume that each vertex in $A$ is adjacent to at most one $A$-ear path in $Q_{2}$. In that case, $G$ is obtained from a multigraph $H$ without loops with $\delta(H) \geq 3$ by subdividing any edge of $H$ at least once and at most twice so that the set of edges of $H$ subdivided twice is independent (in $H$ ). Hence, let $u_{1} v_{1}, \ldots, u_{k} v_{k}$ be the edges of $H$ subdivided twice and let $A^{\prime \prime}$ be the set of all vertices in $H$ for which all edges that are incident are subdivided once. Therefore, we have $|V(H)|=$ $2 k+\left|A^{\prime \prime}\right|$ and $|E(H)|=\frac{1}{2} \sum_{v \in V(H)} \operatorname{deg}_{H}(v) \geq \frac{3}{2}|V(H)|=3 k+\frac{3}{2}\left|A^{\prime \prime}\right|$ (because $\delta(H) \geq 3, k$ edges of $H$ are subdivided twice and the remaining edges are subdivided once). Hence, the order of $G$ is

$$
n=|V(H)|+|E(H)|+k \geq 6 k+\frac{5}{2}\left|A^{\prime \prime}\right| .
$$

Assume that, for each $i$, the edge $u_{i} v_{i}$ in $H$ once subdivided twice produces the path $u_{i} w_{i} z_{i} v_{i}$ in $G$. One can easily see that the function $g$ defined on $V(G)$ by $g(x)=2$ for $x \in V(H), g\left(w_{i}\right)=$ $g\left(z_{i}\right)=1$ for each $1 \leq i \leq k$ and $g(x)=\varnothing$ otherwise is an OIDRDfunction of $G$ such that $g(x) \geq 2$ for each $x \in A$ and

$$
\omega(g)=2|V(H)|+2 k=6 k+2\left|A^{\prime \prime}\right|<\frac{4\left(6 k+\frac{5}{2}\left|A^{\prime \prime}\right|\right)}{3} \leq \frac{4 n}{3} .
$$

This completes the proof. $\square$

Theorem 4.5. If $G$ is a connected $n$-vertex graph with $\delta(G) \geq 2$ such that the set of vertices with degree at least three is independent, then

$$
\gamma_{\text {oidR }}(G) \leq \frac{4 n}{3}
$$

This bound is sharp for $C_{3}$.

Proof. We use an induction on the order $n$. Clearly, $n \geq 3$ since $\delta(G) \geq 2$. If $n \in\{3,4\}$, then $G \in\left\{C_{3}, C_{4}\right\}$ and the result is true by Proposition 4.1, establishing the base cases. Let $n \geq 5$, and assume that the result holds for all graphs $G^{\prime}$ of order less than $n$ with minimum degree at least two such that the set of vertices with degree at least three is independent. Let $G$ be a graph of order $n$ such that $\delta(G) \geq 2$ and the set of vertices with degree at least three is independent. If $\Delta(G)=2$, then $G=C_{n}$ and the result follows from Proposition 4.1. Hence, we assume that $\Delta(G) \geq 3$, and let $A=\left[v \in V(G) \mid \operatorname{deg}_{G}(v) \geq 3\right]$ and $B=V(G)-A$. Consider the $A$-ear paths and keep the same notations as defined in the proof of Proposition 4.4. Note that $A=\cup_{P \in Q} X_{P}$, $V(G)=A \cup \cup_{P \in \mathcal{Q}} V(P)$, and $1 \leq\left|X_{P}\right| \leq 2$ for each $P \in Q$.

Assume first that there exists an $A$-ear path $P$ such that $\delta[G-V(P)]=1$. Since $G$ is simple, this means that $|V(P)| \geq 2$ and some vertex of $G$ of degree three is adjacent to the endvertices of $P$. Thus, $\left|X_{P}\right|=1$. In that case, let $X_{P}=\{a\}$ and $N_{G}(a)-V(P)=\{b\}$. Clearly, $b \in B$ (since $A$ is independent), and thus there is a unique $A$-ear path $P^{\prime}$ in which $b$ is an endvertex of $P^{\prime}$. Let $c$ be the other end-vertex of $P^{\prime}$ (possibly $b=c$ ). Let $G^{\prime}$ be the graph resulting from the deletion of vertex $a$ and all vertices of $P$ and $P^{\prime}$. Then, $\delta\left(G^{\prime}\right) \geq 2$ and by the induction hypothesis, $\gamma_{o i d R}\left(G^{\prime}\right) \leq 4\left|V\left(G^{\prime}\right)\right| / 3$. On the other hand, since $G^{\prime \prime}=G\left[V(P) \cup V\left(P^{\prime}\right) \cup(a)\right]$ is isomorphic to $C_{|V(P)|+1,\left|V\left(P^{\prime}\right)\right|}$, by Proposition 4.3, $G^{\prime \prime}$ has an OIDRD-function $g$ such that $\omega(g) \leq 4 n\left(G^{\prime \prime}\right) / 3$ and $g(c) \geq 1$. Now, for any $\gamma_{\text {oidR }}\left(G^{\prime}\right)$ function, the function $h$ defined on $V(G)$ by $h(x)=f(x)$ for all $x \in V\left(G^{\prime}\right)$ and $h(x)=g(x)$ for all $x \in V\left(G^{\prime \prime}\right)$ is an OIDRDfunction of $G$. Therefore,

$$
\begin{aligned}
\gamma_{\text {oidR }}(G) & \leq \gamma_{\text {oidR }}\left(G^{\prime}\right)+\gamma_{\text {oidR }}\left(G^{\prime \prime}\right) \\
& \leq \frac{4\left|V\left(G^{\prime}\right)\right|}{3}+\frac{4\left|V(P) \cup V\left(P^{\prime}\right) \cup\{a\}\right|}{3}=\frac{4 n}{3} .
\end{aligned}
$$

Next, we can assume that $\delta[G-V(P)] \geq 2$ for each $A$-ear path $P \in \mathcal{Q}$. It follows that $\left|X_{P}\right|=2$ for each $A$-ear path $P \in \mathcal{Q}$. Assume that $\mathcal{Q}-\left(Q_{1} \cup Q_{2} \cup Q_{3} \cup Q_{4} \cup Q_{5}\right) \neq \varnothing$, and let $P \in \mathcal{Q}-\left(Q_{1} \cup Q_{2} \cup Q_{3} \cup Q_{4} \cup Q_{5}\right)$. Note that, by Proposition 4.2, $P$ has an OIDRD-function $g$ such that $\omega(g) \leq 4|V(P)| / 3$ and $g$ assigns positive weight to the end-vertices of the path $P$. Now, let $G^{\prime}$ be the graph obtained from $G$ by removing all vertices of $P$. By the induction hypothesis on $G^{\prime}$, we have $\gamma_{\text {oidR }}\left(G^{\prime}\right) \leq 4\left|V\left(G^{\prime}\right)\right| / 3$. Clearly, for every $\gamma_{\text {oidR }}\left(G^{\prime}\right)$-function $f$, the function $h$ defined on $V(G)$ by $h(x)=f(x)$ for all $x \in V\left(G^{\prime}\right)$ and $h(x)=g(x)$ for all $x \in V(P)$ is an OIDRD-function of $G$, and thus $\gamma_{\text {oidR }}(G) \leq \gamma_{\text {oidR }}\left(G^{\prime}\right)+\gamma_{\text {oidR }}(P) \leq 4 n / 3$. For the remaining part of the proof, we can assume that $\mathcal{Q}=Q_{1} \cup Q_{2} \cup Q_{3} \cup Q_{4} \cup Q_{5}$. Therefore, $G \in \mathcal{F}$, and thus the result follows from Proposition 4.4. $\square$

\section{CONCLUSION}

In this article, we continued the study of outer-independent double Roman domination number and we characterized the trees $T$ of order $n \geq 3$, for which $\gamma_{\text {oidR }}(T) \leq 5 n / 4$, answering a problem posed by Abdollahzadeh Ahangar et al., Ref. 19. Moreover, we showed that, for any connected graph $G$ of order $n$ with minimum degree at least two in which the set of vertices with degree at least three is independent, $\gamma_{\text {oidR }}(G) \leq 4 n / 3$. Finding a sharp upper bound for the outer-independent double Roman domination number of connected graph $G$ of order $n$ with minimum degree remains open.

\section{DATA AVAILABILITY STATEMENT}

The original contributions presented in the study are included in the article/Supplementary Material. Further inquiries can be directed to the corresponding author. 


\section{AUTHOR CONTRIBUTIONS}

All authors listed have made a substantial, direct, and intellectual contribution to the work and approved it for publication.

\section{REFERENCES}

1. Beeler RA, Haynes TW, Hedetniemi ST. Double Roman domination. Discrete Appl Math (2016) 211:23-29. doi:10.1016/j.dam.2016.03.017

2. Abdollahzadeh Ahangar H, Amjadi J, Atapour M, Chellali M, Sheikholeslami SM. Double Roman trees. Ars Combinatoria (2019) 145:173-183.

3. Abdollahzadeh Ahangar H, Amjadi J, Chellali M, Nazari-Moghaddam S, Sheikholeslami SM. Trees with double Roman domination number twice the domination number plus two. Iran J Sci Technol Trans A Sci (2019) 43: 1081-1088. doi:10.1007/s40995-018-0535-7

4. Abdollahzadeh Ahangar H, Chellali M, Sheikholeslami SM. On the double Roman domination in graphs. Discrete Appl Math (2017) 232:1-7. doi:10.1016/ j.dam.2017.06.014

5. Amjadi J, Nazari-Moghaddam S, Sheikholeslami SM, Volkmann L. An upper bound on the double Roman domination number. J Combin Optim (2018) 36: 81-89. doi:10.1007/s10878-018-0286-6

6. Hao G, Volkmann L, Mojdeh DA. Total double Roman domination in graphs. Commun Comb Optim (2020) 5:27-39. doi:10.22049/CCO.2019.26484.1118

7. Jafari Rad N, Azvin F, Volkmann L. Bounds on the outer-independent double Italian domination number. Commun Comb Optim (2021) 6:123-136. doi:10. 22049/CCO.2020.26928.1166

8. Khoeilar R, Chellali M, Karami H, Sheikholeslami SM. An improved upper bound on the double Roman domination number of graphs with minimum degree at least two. Discrete Appl Math (2019) 270:159-167. doi:10.1016/j.dam. 2019.06.018

9. Klobučar A, Klobučar A. Properties of double Roman domination on cardinal products of graphs. Ars Math Contemp (2020) 19:337-349. doi:10.26493/18553974.2022.44a

10. Maimani HR, Momeni M, Nazari-Moghaddam S, Rahimi Mahid F, Sheikholeslami SM. Independent double Roman domination in graphs. Bull Iranian Math Soc (2020) 46:543-555. doi:10.1007/s41980-019-00274-8

11. Volkmann L. The double Roman domatic number of a graph. J Combin Math Combin Comput (2018) 104:205-215.

12. Volkmann L. Double Roman domination and domatic numbers of graphs. Commun Comb Optim (2018) 3:71-77. doi:10.22049/CCO.2018.26125.1078

\section{FUNDING}

This work was supported by the National Key R\&D Program of China (No. 2018YFB1005100).

13. Yue J, Wei M, Li M, Liu G. On the double Roman domination of graphs. Appl Math Comput (2018) 338:669-675. doi:10.1016/j.amc.2018.06.033

14. Zhang X, Li Z, Jiang H, Shao Z. Double Roman domination in trees. Inf Process Lett (2018) 134:31-34. doi:10.1016/j.ipl.2018.01.004

15. Abdollahzadeh Ahangar H, Álvarez MP, Chellali M, Sheikholeslami SM, Valenzuela-Tripodoro JC. Triple Roman domination in graphs. Appl Math Comput (2021) 391:125444. doi:10.1016/j.amc.2020.125444

16. Chellali M, Jafari Rad N, Sheikholeslami SM, Volkmann L. Roman domination in graphs In: TW Haynes, ST Hedetniemi, MA Henning, editors Topics in domination in graphs. Springer International Publishing (2020). doi:10.1007/978-3-030-51117-3

17. Chellali M, Jafari Rad N, Sheikholeslami SM, Volkmann L. Varieties of Roman domination. In: TW Haynes, ST Hedetniemi, MA Henning, editors Structures of domination in graphs. Springer International Publishing (2021). doi:10. 1007/978-3-030-58892-2

18. Chellali M, Jafari Rad N, Sheikholeslami SM, Volkmann L. Varieties of Roman domination II. AKCE Int J Graphs Comb (2020) 17:966-984. doi:10.1016/j. akcej.2019.12.001

19. Abdollahzadeh Ahangar H, Chellali M, Sheikholeslami SM. Outer independent double Roman domination. Appl Math Comput (2020) 364: 124617. doi:10.1016/j.amc.2019.124617

20. Mojdeh DA, Samadi B, Shao Z, Yero IG. Outer independent double Roman domination number of graphs. Appl Math Comput (2020) 364:124617. doi:10. 1016/j.amc.2019.124617

Conflict of Interest: The authors declare that the research was conducted in the absence of any commercial or financial relationships that could be construed as a potential conflict of interest.

Copyright (c) 2021 Rao, Kosari, Sheikholeslami, Chellali and Kheibari This is an open-access article distributed under the terms of the Creative Commons Attribution License (CC BY). The use, distribution or reproduction in other forums is permitted, provided the original author(s) and the copyright owner(s) are credited and that the original publication in this journal is cited, in accordance with accepted academic practice. No use, distribution or reproduction is permitted which does not comply with these terms. 